\title{
External parasitic elements on clothing for improved performance of wearable antennas
}

\author{
M.Vatankhah Varnoosfadeani, Student Member, IEEE, David V. Thiel, Senior Member, IEEE, \\ Junwei Lu, Senior Member, IEEE
}

\begin{abstract}
A small (30 mm x 50mm x $8 \mathrm{~mm})$, waterproof 2.45 GHz wireless sensor with a standard meander line printed monopole has poor radiation characteristics when placed against the human body. A suitably optimized $\mathrm{H}$-shape (step impedance resonator) parasitic element was added next to the antenna of the sensor on outer surface of clothing fabric. The parasitic element was placed on the outside of a fabric armband and was found to increase the total efficiency of the antenna by $13 \%$ in simulation results and enhanced the measured gain by $3 \mathrm{~dB}$. The technique ensures the armband is comfortable, washable, flexible, and operates successfully without wired connectors. The technique was optimized using simulation and experimental measurements with an adhesive aluminum strip. As the wireless sensor is waterproof and there is no access to the port of the antenna in the sensor, a two ray ground reflection model was used to match the received power in the spectrum analyzer using least RMS curve fitting.
\end{abstract}

Index Terms - wearable antennas, clothing, parasitic elements, wireless sensors, two ray ground reflection model

\section{INTRODUCTION}

I NTEREST in body-worn wireless sensors has increased in recent years because of their wide applications in sports, mining, navigation, tracking, health-care etc. One of the major problems in body worn wireless sensors is the negative effect of human body on the performance of device's antenna which causes a significant decrease in the signal strength and range of communication which confines the applications of the sensor [1-4]. Matching circuits play an important role in structure of wireless sensors to match the input impedance of the antenna and output impedance of transceiver IC [4] because in some cases the output impedance is designed for a balanced antenna or it is not $50 \Omega$ [5]. Matching circuits are designed for free space conditions and when the antenna is placed next to human tissues, the input impedance of the antenna drastically changes. In addition, a huge proportion of power would be absorbed in body tissues[3]. Body absorption causes $13 \sim 16.2 \mathrm{dBm}$ attenuation in

Manuscript received February, 2014; revised July 2014; accepted July 2014 Authors are with the Centre for Wireless Monitoring and Applications, Griffith University, Brisbane, QLD , Australia. (e-mail: d.thiel@griffith.edu.au ). "Copyright (c) 2013 IEEE. Personal use of this material is permitted. However, permission to use this material for any other purposes must be obtained from the IEEE by sending a request to pubs-permissions@ieee.org." the received signal strength at $2.4 \mathrm{GHz}$ [6]. In [7], the effects of size of the ground plane and the distance between the ground plane and the human body were investigated to present a possible solution for avoiding power absorption in body tissues, but the required size of the antenna would increase to more than two times bigger than the sensor itself. Wearable fabric antennas became popular in recent years because of their flexibility and ease of attachment to garment. The idea of circuits on fabric appeared recently $[8,9]$, but only some simple circuits are presented and this technology was not used for fabrication of wireless nodes. Robust long-term connection between the rigid electronic components of the sensor and the textile antennas is a significant problem. This has limited the usage of fabric antennas for wearable wireless networks [10]. The use of coaxial cables and connectors and rigid and semi-rigid structures provide unyielding surfaces and edges and so cause major discomfort to the user. Cables and connectors are also quite unreliable given the continued flexing of the fabric. Moreover, in sport applications on the playing field, it is not easy for a player to wear a sensor with bulky SMA connectors and cables. This paper proposes a method to improve the performance of the wireless sensor by adding a parasitic element next to the antenna of the sensor. In this way, a kind of wearable antenna with simple connectivity and without any connector has been introduced. The parasitic element, using electromagnetic near field coupling [11] matches the input impedance of the antenna to the transmitter at $2.45 \mathrm{GHz}$ and compensates the effect of the body shift in resonant frequency. Furthermore, it shapes the radiation pattern to the direction outside of the body and increases the gain of the antenna to improve signal strength at a receiver [12]. To investigate the effects of the parasitic element on the antenna and to find the best size, position and orientation of the parasitic element, a series of simulation and optimization steps were undertaken using a commercial FEM package [13]. A step impedance resonator (H- shape) has been chosen as the parasitic element to have several degrees of freedom in the optimization process. MATLAB [14] was used to analyze a two ray ground reflection model to calculate the received power in receiver. The mean square error between the measured and calculated data to fit a curve on measured data on a grassed sports field was used to quantify the improvement in transmitted power.

\section{WIRELESS SENSOR}

The Centre for Wireless Monitoring and Applications at Griffith University has developed small wireless sensor nodes to monitor human movement in sport during both training and 
match play[15]. The sports employing these techniques include football, swimming, tennis, running, field hockey, boxing and many others. One device (z-core [5]) consists of a 3 axis accelerometer, a three axis gyroscope, Wi-Fi connectivity (2.45 $\mathrm{GHz}$ ), a USB rechargeable battery and a colored LED display (Figure.1.a). The double layered FR4 printed circuit board in the sensor includes a standard design [16] meander line monopole antenna (Figure.1.b). The device is housed in a robust rectangular plastic case measuring $50 \mathrm{~mm} \times 30 \mathrm{~mm}$ x $8 \mathrm{~mm}$. The device is usually worn in a fabric armband with Velcro fastening with a specially designed pouch to allow simple installation before and removal after use. (Figure.2.a)

While the free space range exceeds $20 \mathrm{~m}$, the range when worn on the arm was reduced to less than $3 \mathrm{~m}$. The decrease in communication range is attributed to the effect of the human body in changing the resonant frequency of the antenna, changing in the radiation pattern, and the absorption of the power by body tissues.

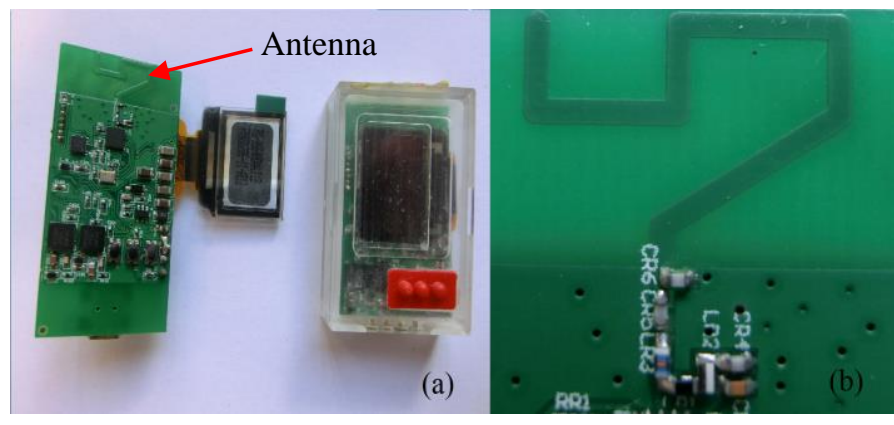

Figure 1.a) Z-core device with and without housing b) Standard design monopole antenna

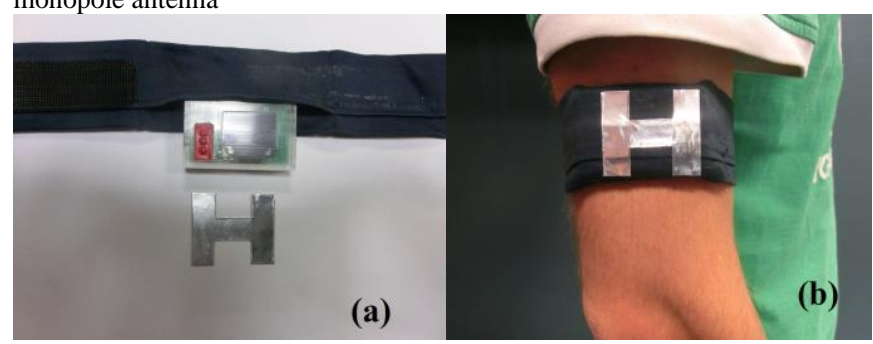

Figure 2.a) Fabric armband and the method of installation of sensor b) parasitic element which is added next to the antenna of the sensor

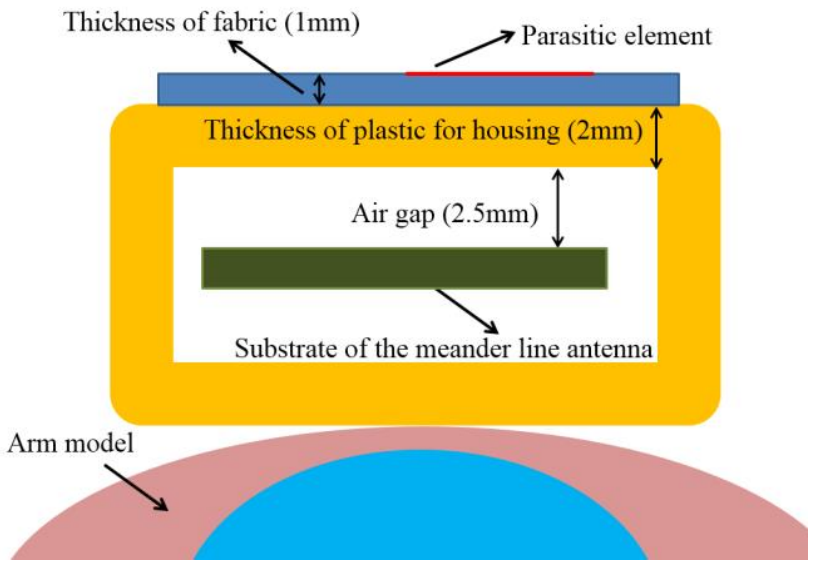

Figure 3.Cross section of the Z-core sensor showing the layers between the parasitic element and the meander line monopole antenna on the arm.

\section{PARASITIC ELEMENT DESIGN AND OPTIMIZATION}

In [12] a coaxial monopole acts as a driven element for an outer parasitic element to improve the gain and match the input impedance of the antenna. Here the same near-field coupling is used [12] as the printed meander line monopole drives an outer parasitic element to shape the radiation pattern to the direction outside of the body and match the input impedance of the antenna and bring the resonant frequency back to $2.45 \mathrm{GHz}$. Given that the driven element is linearly polarized, a simple straight parasitic element was placed parallel to the major axis of the meander line monopole and separated from the PCB by a $5.5 \mathrm{~mm}$ distance. This distance is the sum of an air gap $(2.5 \mathrm{~mm})$, the thickness of plastic housing $(2 \mathrm{~mm})$ and the fabric thickness $(1 \mathrm{~mm})$ which is depicted in figure.3. The structure under the parasitic element is a stacked combination of fabric, plastic and air which are placed next to the arm. As a consequence, it is complicated to calculate the effective permittivity and length of the parasitic element to be half of a wavelength at $2.45 \mathrm{GHz}$. This parasitic element was added to compensate for the effect of the arm on the performance of the antenna. Using CST Microwave an optimization started with a simple straight parasitic element for the model presented in Figure 3 to minimize S11 and maximize gain at $2.45 \mathrm{GHz}$ by changing the length, width and position of parasitic element on the top of the meander line monopole (figure.4). Simple straight line parasitic element confines the optimization process because there are just three parameters of length, width and position of parasitic element next to the meander line antenna which can be optimized. As a result the input impedance of the meanderline antenna cannot be perfectly matched at $2.45 \mathrm{GHz}$ (figure.5). Moreover, the shape and direction of radiation pattern changes by sweeping the position of straight line parasitic element in XY plane and in X direction (figures 4 and 6). In figure 6, varying the position of parasitic element in $\mathrm{X}$ direction in $\mathrm{XY}$ plane changes the shape and direction of radiation pattern in $\mathrm{XZ}$ plane because of electromagnetic coupling mentioned in [12]. In figure.7, parasitic element was swept in $\mathrm{X}$ direction again but the $\mathrm{YZ}$ plane was monitored (along with axis of meander line antenna) and the shape and direction of pattern in YZ plane did not change significantly. As a consequence of the two parametric simulations in figures 6 and 7, the straight line parasitic element is sensitive to possible movements of parasitic element in the $\mathrm{X}$ direction during field measurements. As a consequence, the straight line parasitic element was replaced with a step impedance resonator because it has two different characteristic impedances which are shown as $Z_{1}$ and $Z_{2}$ in figure 4 . This provides the optimization process with several parameters and more degrees of freedom to match the input impedance of the antenna at $2.45 \mathrm{GHz}$ with $\mathrm{H}$ shaped parasitic element and the sensitivity of return loss to unpredicted movements of parasitic element is less than the straight line parasitic element (figure. 8). Moreover, the shape and direction of the pattern did not vary by changing the position of H-shape parasitic element (Figures 9, 10). Finally, a H-shape parasitic element was considered rather than a simple straight $\lambda / 2$ parasitic element to have a better input impedance matching and less sensitivity to possible movements of parasitic element. 
The optimization for the H- shape parasitic element had six different parameters $\mathrm{W}, \mathrm{W}_{\mathrm{s}}, \mathrm{P}, \mathrm{L}_{1}, \mathrm{~L}_{2}$ and $\mathrm{L}_{3}$ to maximize the gain and minimize the S11 at $2.45 \mathrm{GHz}$. The optimized dimensions of parasitic element are presented in table 1 . An adhesive aluminum sheet (Tesa ${ }^{\circledR}$ 50565) which is moisture resistant with the thickness of $50 \mu \mathrm{m}$ was used to fabricate the parasitic element. A parametric study was done to compare the sensitivity of radiation pattern and impedance matching of meander line antenna to the position of $\mathrm{H}$-shape parasitic element. In this way, effects of possible movements of parasitic element during field measurements can be predicted and considered. The sensitivity of the radiation pattern and return loss to the position of the parasitic element and the changes in the peak gain of the whole structure is higher for a simple straight line compared to the H-shaped element (figures 7-10). Moreover, the linear straight parasitic element cannot perfectly match the input impedance of the antenna at $2.45 \mathrm{GHz}$ as the $\mathrm{H}$-shape parasitic element does.

The distance between the major axis of the meander line antenna and the major axis of parasitic elements in figure 4 shown by parameter $P$ was changed between -15 to $15 \mathrm{~mm}$ and that causes parasitic element was swept in $\mathrm{x}$ direction. This is a possible movement while the armband is worn on the arm. Negative $\mathrm{P}$ is when the major axis of a parasitic element is at the right side of the major axis of the meander line antenna (figure.4). The optimized value for $\mathrm{P}$ is 9.09 as is mentioned in table 1 . Some other values of $P$ are selected between -15 and 15 to show sensitivity of parasitic element to possible movements during field measurements. The parametric study is presented in figures (5-10) and shows the changes in radiation pattern and return loss of the antenna when the parasitic elements (straight line and $\mathrm{H}$-shape) move in $\mathrm{XY}$ plane. The radiation pattern in $\mathrm{XZ}$ plane is sensitive to the movements of straight line parasitic element and even the direction of peak gain of the pattern changes in this plane by sweeping the parasitic element along with the arm in $\mathrm{x}$ direction. The gain changes between -10 and 0.2 in YZ plane (figure 7) for straight line parasitic element. In $\mathrm{XZ}$ plane for straight line the gain changes between -10 and 1.1 and the direction of peak gain can have 30 degrees deviation from the normal direction (Figures 6,7).The peak gain for $\mathrm{H}$ shape parasitic element changes between -7.5 and 1.8 in both XZ and YZ planes ( Figures 9,10).

TABLE 1: Optimized parameters of the parasitic element shown in Figure. 4 (All dimensions are in $\mathrm{mm}$ )

\begin{tabular}{|c|c|c|c|c|c|}
\hline $\mathrm{W}$ & $\mathrm{W}_{\mathrm{s}}$ & $\mathrm{P}$ & $\mathrm{L}_{1}$ & $\mathrm{~L}_{2}$ & $\mathrm{~L}_{3}$ \\
\hline 33.4 & 11 & 9.09 & 13.7 & 11.2 & 18.6 \\
\hline
\end{tabular}

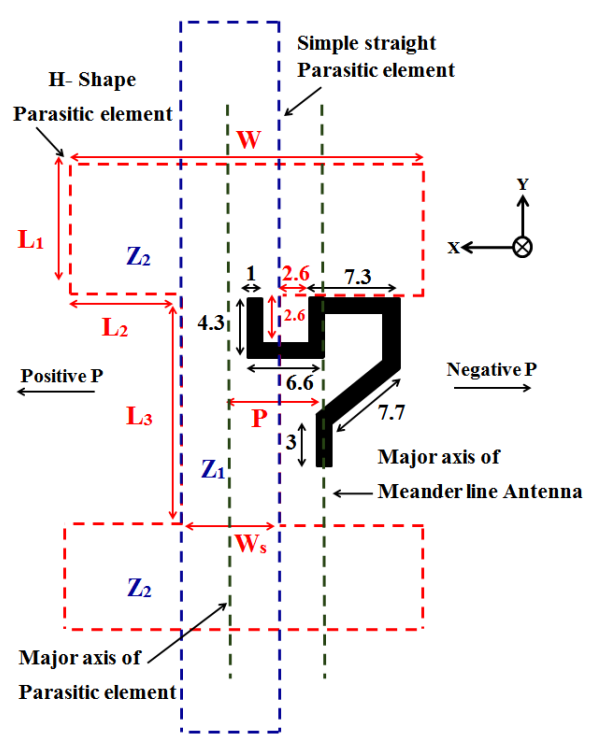

Figure 4.Dimensions of the antenna and the optimized position of the parasitic element $(\mathrm{mm})$

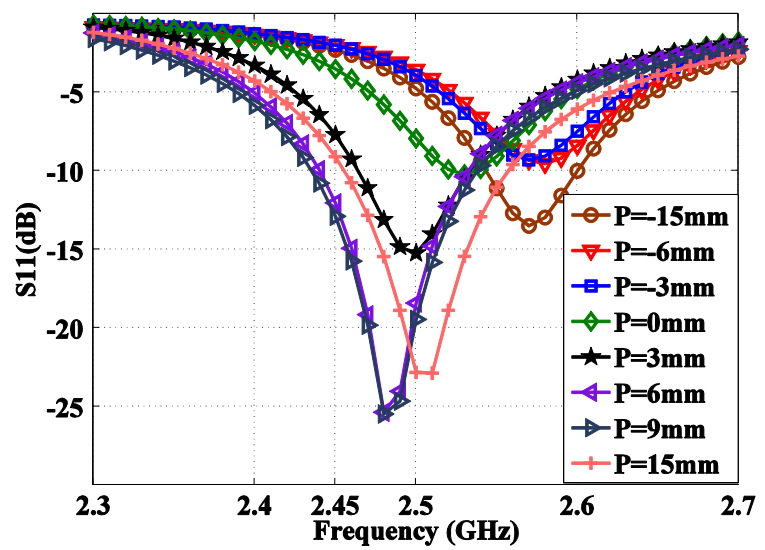

Figure 5.Changes in simulated return loss of antenna by changing the position of simple straight parasitic element about major axis of meander line antenna.

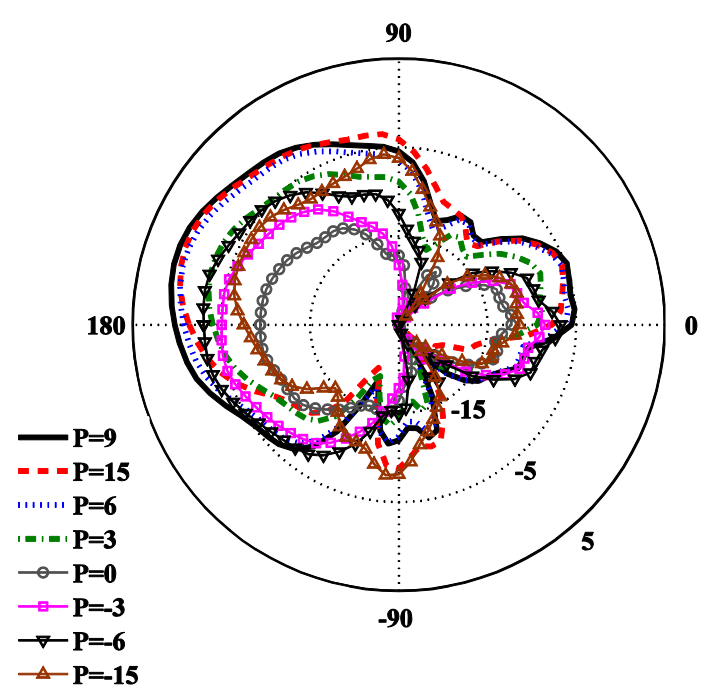

Figure 6.Changes in simulated realized gain of antenna by changing the position of simple straight parasitic element about major axis of meander line antenna (XZ plane). 


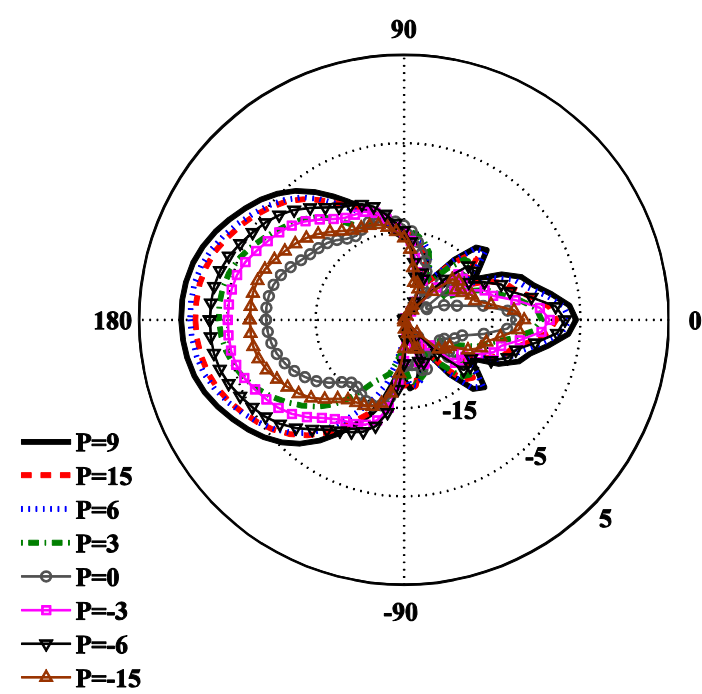

Figure 7.Changes in simulated realized gain of antenna by changing the position of simple straight parasitic element about major axis of meander line antenna (YZ plane)

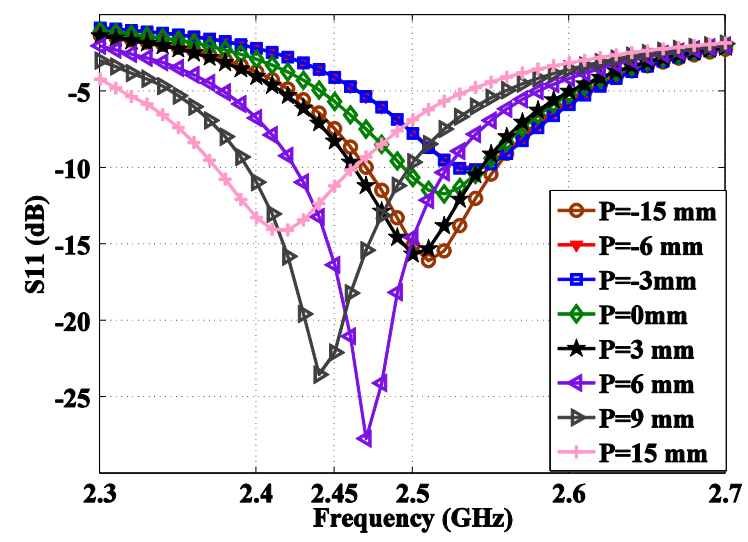

Figure 8.Changes in simulated return loss of antenna by changing the position of $\mathrm{H}$-shape parasitic element about major axis of meander line antenna.

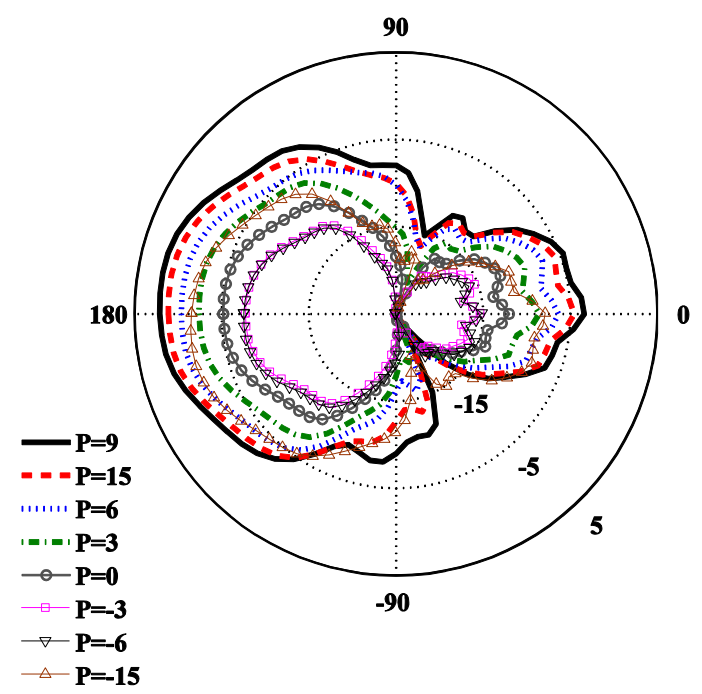

Figure 9.Changes in simulated realized gain of antenna by changing the position of $\mathrm{H}$-shape parasitic element about major axis of meander line antenna (XZ plane).

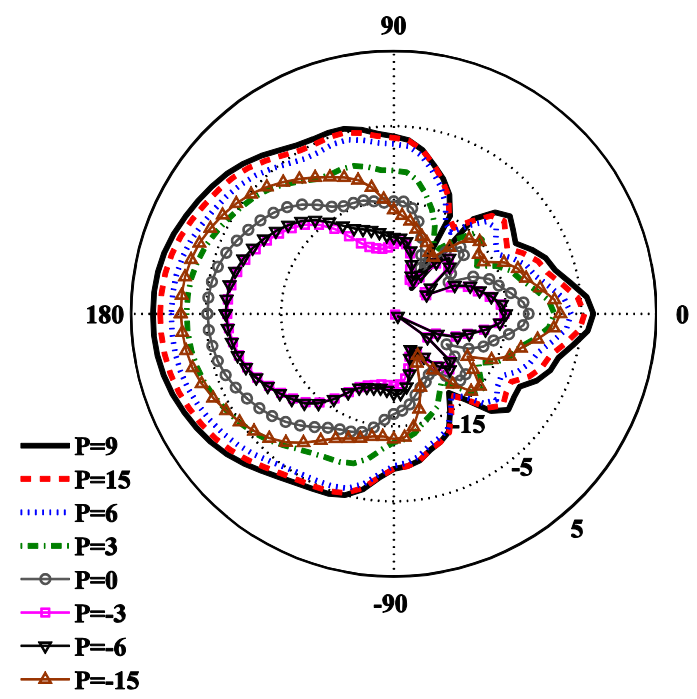

Figure 10.Changes in simulated realized gain of antenna by changing the position of $\mathrm{H}$-shape parasitic element about major axis of meander line antenna (YZ plane).

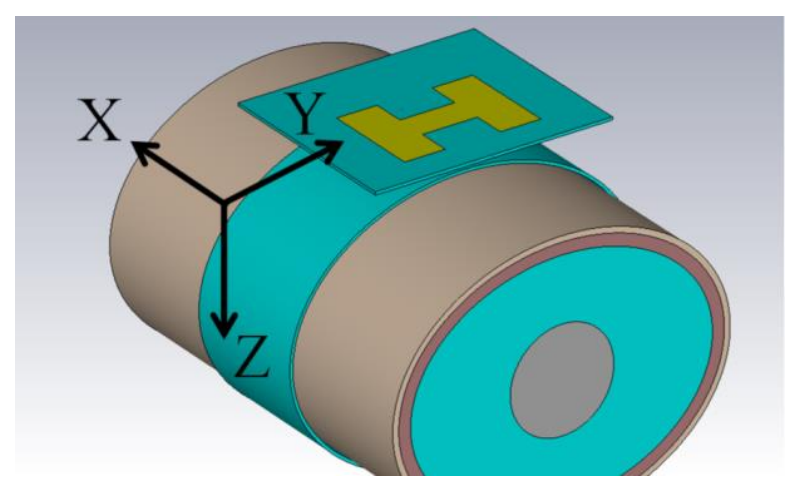

Figure 11.Simulated antenna next to the arm model in CST Microwave Studio.

\section{SIMULATION RESULTS}

TABLE 2: Dielectric properties of different parts of arm model at $2.45 \mathrm{GHz}$

\begin{tabular}{|c|c|c|c|c|}
\hline $\begin{array}{c}\text { Dielectric } \\
\text { properties }\end{array}$ & Skin & Fat & Bone & Muscle \\
\hline Permittivity & 38.06 & 5.28 & 18.6 & 52.79 \\
\hline Loss tangent & 0.28 & 0.14 & 0.31 & 0.24 \\
\hline
\end{tabular}

To simulate the effects of the arm on the performance of the antenna, an arm model recommended by [17] was used (fig.11).It includes four layers of $2 \mathrm{~mm}$ skin thickness , $4 \mathrm{~mm}$ fat thickness, $17.5 \mathrm{~mm}$ bone radius, centered $4 \mathrm{~mm}$ away from the elliptical shape center and the muscle fills the space between fat and bone. The dielectric properties of different parts of the arm at 2.45 $\mathrm{GHz}$ are presented in table 2.[18].

Furthermore, two layers of fabric with the thickness of $1 \mathrm{~mm}$ used beneath and on the top of the plastic case simulate the fabric armband. The permittivity and loss tangent of fabric are 1.63 and 0.013 respectively. Based on Table 1 in [1], the total radiation 
efficiency of the antenna is a good criterion to explain the performance of the antenna next to the body because this includes the mismatch and the body absorption together. This can be written as (1):

$$
\text { Total radiation efficiency }=\frac{R_{r}}{R_{r}+R_{l}} \times\left(1-|\Gamma|^{2}\right)
$$

Fig. 12 shows the variation in resonant frequency of the antenna before and after locating the box on the body. Fig. 13 shows the total efficiency of the simulated antenna in the free space, in the box and next to the arm model with and without the parasitic element. With the parasitic, the total radiation efficiency of the antenna improved by $13 \%$ at $2.45 \mathrm{GHz}$. This is the result of a shift in the resonant frequency and a reduction in the power absorption. Although the parasitic element has an effect on the resonant frequency of the antenna, it is also expected to change the pattern and shape the radiation pattern. The radiation pattern of the antenna was investigated using CST Microwave Studio to model the antenna on the arm. Figures 14 and 15 show the simulated patterns with and without the parasitic element. Adding the parasitic element, the peak gain of the antenna improved by $1.9 \mathrm{~dB}$ and shaped the pattern in the direction away from the body.

\section{MEASUREMENT}

The sensor is waterproof and the access to the port of the antenna is not possible. Two measurement methods were used to investigate the effects of parasitic element on the performance of the antenna. In the first method, the power pattern of the sensor was measured with and without the parasitic element on the body. Fig. 16 shows the measurement setup which includes a wooden tripod $(160 \mathrm{~cm}$ height), a RF explorer handheld spectrum analyzer [19] model $2.4 \mathrm{G}$ and a wooden rotator. The power measurement resolution for RF explorer is $0.5 \mathrm{dBm}$ which is enough for desired measurements. A female person $(156 \mathrm{~cm}$ height, $52 \mathrm{Kg}$ weight) was wearing the sensor on her arm on the height of $112 \mathrm{~cm}$ from the ground). The distance between the rotator and the tripod was fixed to $3 \mathrm{~m}$ to ensure adequate signal strength in receiver. The signal strength was measured at 15 degree intervals through 360 degrees. The body was located offcenter on the rotator to make sure that the antenna is in the middle of the rotator and the separation distance between the transmitter and receiver did not change. The signal strength was measured in $\mathrm{dBm}$. The maximum signal strength was normalized to $0 \mathrm{dBm}$ (see figure 17). The experiments were conducted on a flat, grassed sports field. Fig.17 shows the results of the first measurement method and Fig.18 shows the schematic for the position of the body, the spectrum analyzer and the antenna on the arm. The measured results are similar to the simulated radiation pattern in CST. The maximum gain of the antenna increased by $3 \mathrm{~dB}$ and the peak power direction is rotated through about 15 degrees with the parasitic element. This effect can be explained as using a body rather than an isolated arm in the experiments, and other factors not considered in simulation such as small changes in the position of the parasitic element and inaccuracies in fabrication. To improve the accuracy and to be able to compare the measurement with simulation results, a two ray ground reflection simulation model (fig.14) for the propagation at the oval was analyzed using MATLAB.

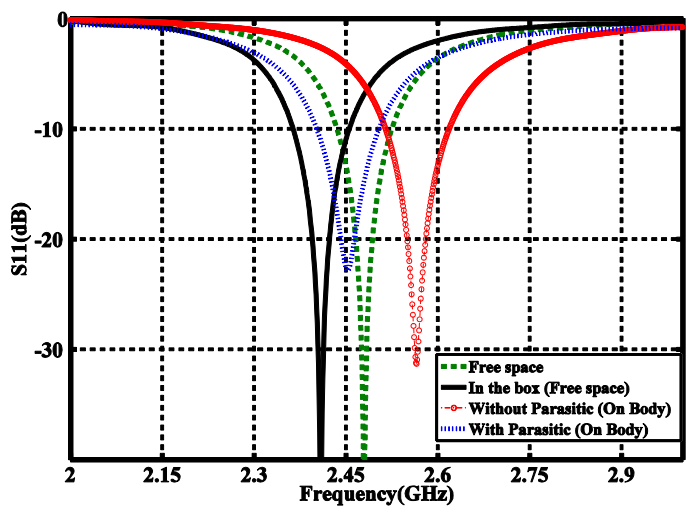

Figure 12.Simulated return loss in free space, in the box (in free space), without parasitic element (on arm model) and with parasitic element (on arm model).

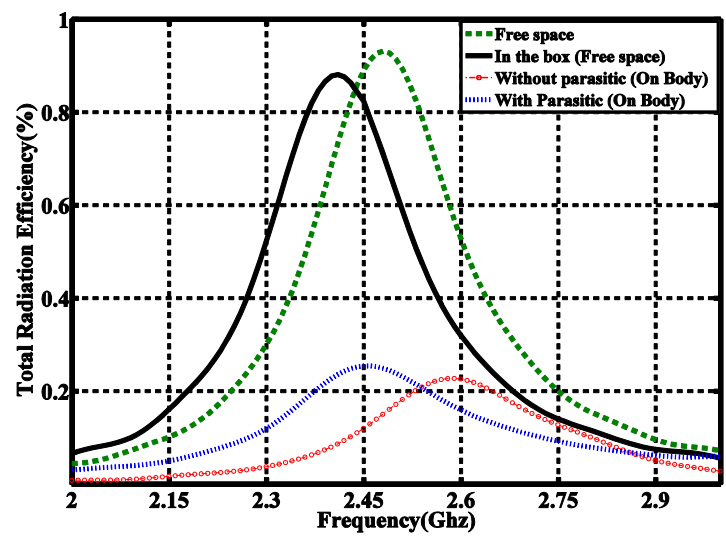

Figure 13. Simulated total radiation efficiency in free space, in the box (in free space), without parasitic element (on arm model) and with parasitic element (on arm model).

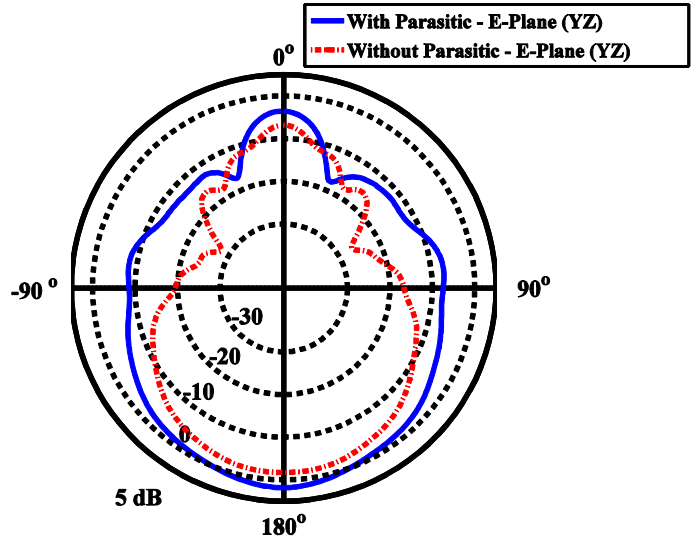

Figure 14.Simulated radiation pattern of the antenna next to the arm model with and without parasitic element (YZ- plane) 


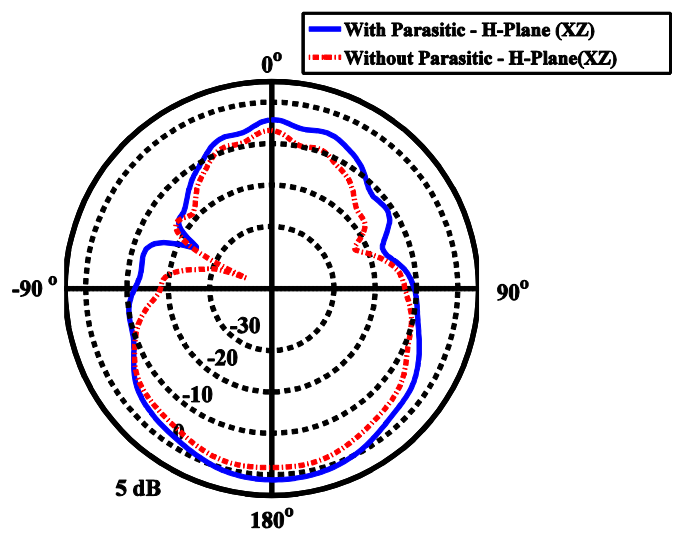

Figure 15.Simulated radiation pattern of the antenna next to the arm model with and without parasitic element (XZ- plane)

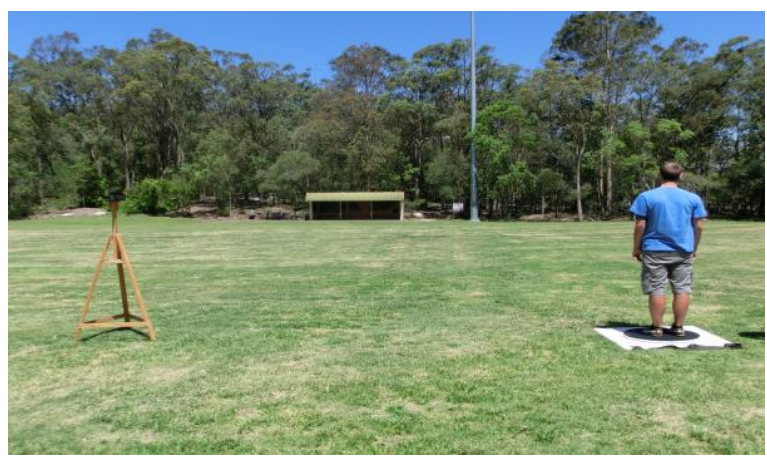

Figure 16.Setup for the first measurement method

In this model, the received power was calculated using a direct ray and a ground reflected ray using equations 2,3 and 4 . The electric field at the receiver from the direct ray path is given by [20]:

$$
E_{\text {direct }- \text { path }}=E_{0}\left|f_{1}\left(\theta_{1}\right) f_{2}\left(\theta_{1}^{\prime}\right) \frac{e^{-j k_{0} R_{1}}}{4 \pi R_{1}}\right|
$$

The ground reflected ray path is given by [20]:

$$
E_{\text {reflected }- \text { path }}=E_{0}\left|f_{1}\left(\theta_{2}\right) f_{2}\left(\theta_{2}^{\prime}\right) \rho e^{j \phi} \frac{e^{-j k_{0} R_{2}}}{4 \pi R_{2}}\right|
$$

$f_{1}$ and $f_{2}$ are factors for involving radiation patterns of the two antennas, $R_{1}$ is the direct distance between receiver and transmitter, $\theta_{1}$ and $\theta_{1}^{\prime}$ are the angles which the direct path makes with horizontal plane at the transmitter and receiver respectively, and $k_{0}$ is the wave number in free space. $R_{2}$ is the reflection distance between receiver and transmitter, $\theta_{2}$ and $\theta_{2}^{\prime}$ are the angles which reflection path makes with horizon in transmitter and receiver respectively.

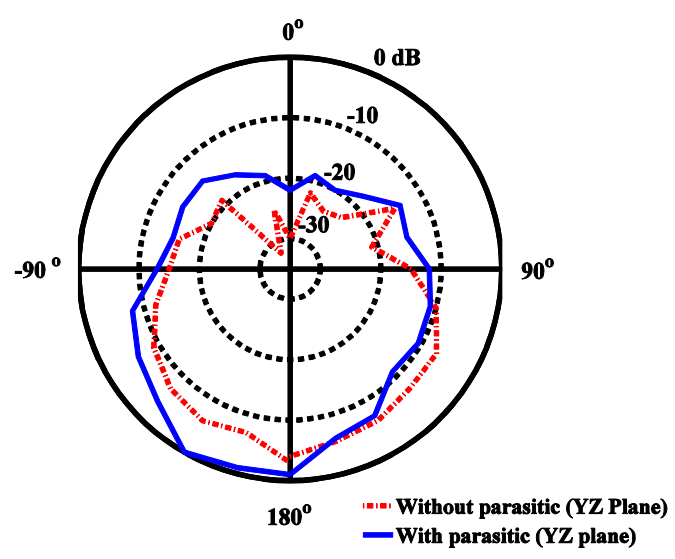

Figure 17.Normalized measured power pattern (relative $\mathrm{dB}$ ) of the sensor with (blue line) and without parasitic element (red line). (YZ plane)
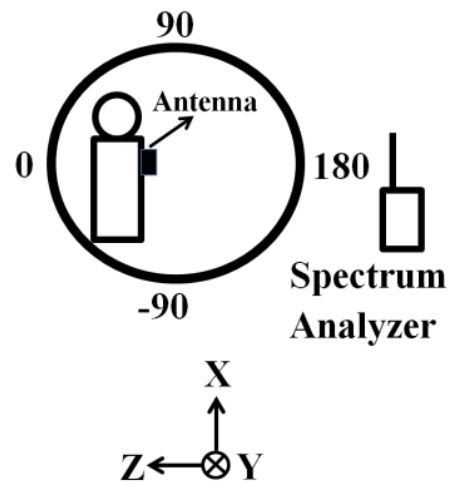

Figure 18.Schematic plan of the setup used in the first measurement method (The antenna is located at the center for the circular rotation platform)

TABLE 3: Optimized outputs

\begin{tabular}{|c|c|c|c|c|c|}
\cline { 2 - 6 } \multicolumn{1}{c|}{} & $\begin{array}{c}\text { RMS } \\
\text { error }\end{array}$ & $\varepsilon_{r}$ & $\sigma(s / m)$ & $E_{0}(V / m)$ & $E_{0}(d B m)$ \\
\hline $\begin{array}{c}\text { With } \\
\text { parasitic } \\
\text { element }\end{array}$ & 24.9 & 7.1 & 0.295 & $8.7 \mathrm{e}-4$ & -61.21 \\
\hline $\begin{array}{c}\text { Without } \\
\text { Parasitic } \\
\text { element }\end{array}$ & 22.8 & 7.1 & 0.307 & $7 \mathrm{e}-4$ & -63.09 \\
\hline
\end{tabular}

The reflection coefficient $\rho e^{-j \varphi}$ is given by the Fresnel expression for vertical polarization. This is given by:

$$
\rho e^{-j \varphi}=\frac{\left(\varepsilon_{r}-j \frac{\sigma}{\omega \varepsilon_{0}}\right) \sin (\psi)-\sqrt{\left(\varepsilon_{r}-j \frac{\sigma}{\omega \varepsilon_{0}}\right)-\cos ^{2}(\psi)}}{\left(\varepsilon_{r}-j \frac{\sigma}{\omega \varepsilon_{0}}\right) \sin (\psi)+\sqrt{\left(\varepsilon_{r}-j \frac{\sigma}{\omega \varepsilon_{0}}\right)-\cos ^{2}(\psi)}}
$$

Where $\varepsilon_{r}$ and $\sigma$ are the permittivity and the conductivity of the ground respectively, $\psi$ is the grazing angle of the incidence, $\varepsilon_{0}$ is the permittivity in free space and $\omega$ is the angular frequency. 
The received power can only be calculated by curve fitting the unknown parameters like the permittivity of the ground, the conductivity of the ground, $f_{1}$ and $f_{2}$ (the radiation patterns of the two antennas). The function $\mathrm{f} 1$ is a factor in the formula which describes the radiation pattern of antenna when the sensor is placed next to the body. The function $f_{2}$ is the radiation pattern of the antenna of the spectrum analyzer. This is a simple monopole so the function $\operatorname{Cos}(\theta)$ was used to approximate the radiation pattern factor of the receiver. The objective is to calculate the transmitted power from antenna of the sensor.

A curve was fitted on the measured radiation pattern of the antenna using calculations in MATLAB (Fig. 20) to be used as $f_{2}$ in MATLAB analysis. In figure 19 , for $1 \leq \mathrm{d} \leq 20, \mathrm{~h}_{1}=1.12 \mathrm{~m}$ and $\mathrm{h}_{2}=1.6 \mathrm{~m}, \theta_{2}$ changes between 7 and 69 degrees. As incidence angle equals the reflection angle then $\psi \approx \theta_{2}$. Thus $\psi$ changes between 7 and 69 degrees. The next step was to find the conductivity and permittivity of the ground covered with grass. To estimate these parameters, 20 distance measurements were used to calculate the unknown parameters using curve fitting based on the least RMS error. The distance between the transmitter and the receiver was increased from 1 to 20 meters with a step size of 1 meter. At each step, the received power was recorded with and without the parasitic element. Figure 21 shows the recorded data and the two ray path model fitted to this data. The inaccuracy in installing of the parasitic element and moving of the sensor in the fabric pouch can change the direction of the peak gain of the radiation pattern. As a consequence, in each step, the maximum received power in the spectrum analyzer was recorded by rotating the body to find the peak gain. An optimization function in MATLAB was defined to accept four matrices of the parameters of conductivity and permittivity of the ground, transmitted power from sensor and measured electrical field strength. The mean square error between the measured data and the calculated data from formulas 2, 3 and 4 was the criteria to find the optimum values to fit the curve and so to find the unknown parameters. Outputs from the MATLAB optimization function were $\varepsilon_{r}, \sigma$ and $E_{0}$. The transmitted power $E_{0}$ from the sensor is obtained. As expected, after optimization, the values of $\varepsilon_{r}$ and $\sigma$ were approximately the same for both states (with and without the parasitic element) while $E_{0}$ is expected to be different with the addition of the parasitic element. By entering these outputs to the two ray reflection model, the simulation results for the electrical strength field in receiver can be compared with measured data (figure 21). The optimization was run for both states independently. Table 3 presents the outputs of the optimization routine.

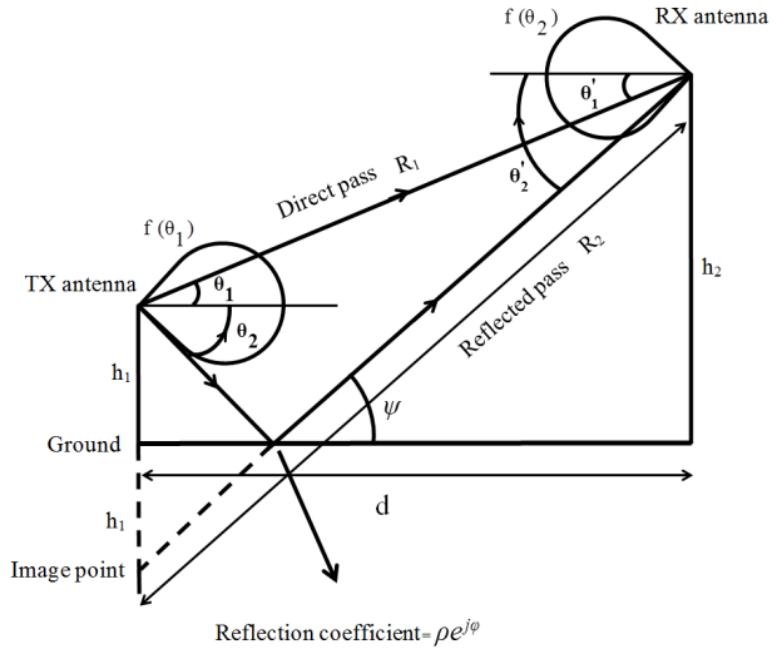

Figure 19.Two ray ground reflection model for vertical polarization.

This increase in the received power is $1.9 \mathrm{~dB}$ which agrees with the CST results. Both curve fits yielded the same values for $\varepsilon_{r}$ and $\sigma$ as expected. Table 4 shows the transmitted half power beam width (HPBW) in H-plane and E-plane with and without the parasitic element.

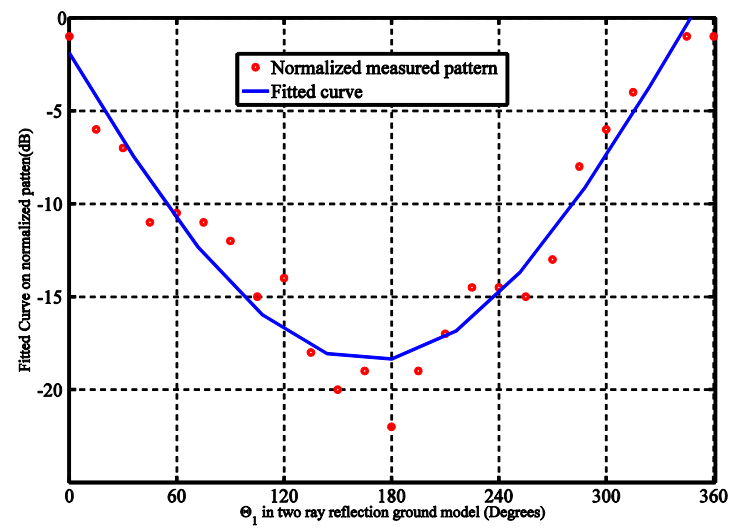

Figure 20. Fitted curve on measured gain of the antenna to calculate $f_{I}$ in equations 3,4 and 5 .

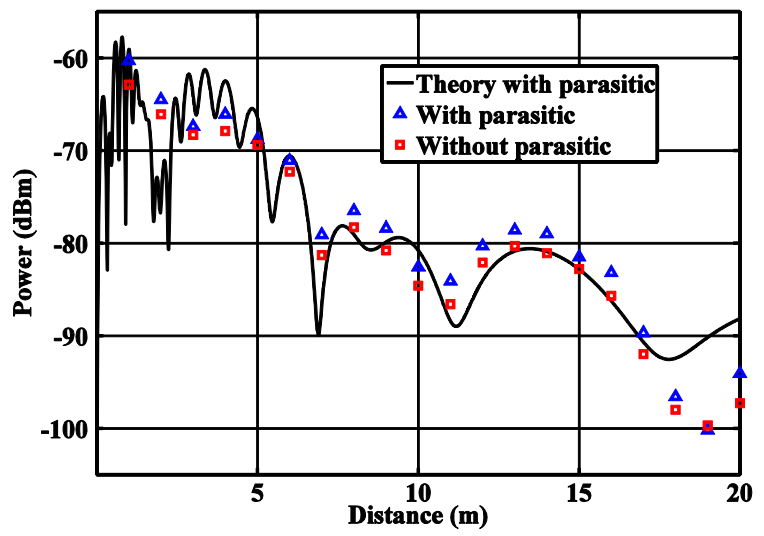

Figure 21.Comparison of calculated power with two ray reflection model and measured power in the oval. 
TABLE 4: Changes in the HPBW (degrees) in measurement and simulation.

\begin{tabular}{|l|l|l|}
\hline HPBW (degree) & H-plane & E-plane \\
\hline With parasitic (CST) & 69.5 & 85.3 \\
\hline Without Parasitic (CST) & 80.9 & 76.9 \\
\hline $\begin{array}{l}\text { Fitted Curve on measured Data } \\
\text { With Parasitic }\end{array}$ & 63 & - \\
\hline $\begin{array}{l}\text { Fitted Curve on measured Data } \\
\text { Without Parasitic }\end{array}$ & 82 & - \\
\hline
\end{tabular}

The variations in gain can be calculated using the measured and simulated values given in table 4 . Let $D$ be the directivity of the antenna, $\Omega_{A}$ is the half-power solid angle and $\beta$ is the HPBW in radians for each of the $\mathrm{xz}$ and $\mathrm{yz}$ planes in the radiation pattern. $G_{p}$ is the gain for the antenna with the parasitic element and $G_{w p}$ is the gain of the antenna without parasitic element. $\beta_{p-H p l a n e}$ is the HPBW in H-plane after adding parasitic element. The relationship between beam width and gain can be written

$$
D=\frac{4 \pi}{\Omega_{A}} \cong \frac{4 \pi}{\beta_{x z} \beta_{y z}} \rightarrow \frac{G_{p}}{G_{w}} \cong \frac{\beta_{w p-\text { Hplane }}}{\beta_{p-\text { Hplane }}} \frac{\beta_{w p-\text { Eplane }}}{\beta_{p-\text { Eplane }}}
$$

Calculations show that the gain increases by $1.33 \mathrm{~dB}$. The difference between this result and the CST simulation can be explained by the effect of changes in radiation efficiency at 2.45 $\mathrm{GHz}$ after adding the parasitic element. This point proves that the improvement in the total efficiency is because of both impedance matching and a reduction in the power absorbed by the body. The CST simulation and the two ray ground reflection model show that the net result is an increase in gain of $1.9 \mathrm{~dB}$ and the radiation pattern measurement presents $3 \mathrm{~dB}$ improvement in gain of the antenna.

\section{CONCLUSION}

A H-shape parasitic element was worn on a fabric armband next to the antenna of a wireless sensor to improve the performance of the whole system by affecting the input impedance and tuning the resonant frequency of the antenna. This parasitic element is an external wearable antenna without connector which improves the performance of the sensor by using near field coupling to feed the wearable antennas rather than bulky SMA connectors or baluns. There is no need to use semi rigid and rigid cables on the body to feed the antenna. The efficiency of the antenna improved by $13 \%$ in the simulation results at $2.45 \mathrm{GHz}$ and peak of power radiation pattern of the sensor increased by $3 \mathrm{~dB}$ in the measurement results. A two ray reflection model from the ground was used to calculate the received power by a spectrum analyzer and the results verify the improved radiation performance of the antenna by $1.9 \mathrm{~dB}$ which agrees with CST results and is less than the power pattern measurement. To separate the role of impedance matching and power absorption, the improvement in gain was calculated using the HPBW. The difference between the calculated and simulated improvement is explained by the change in the radiation efficiency as there is less power absorbed by the body.

\section{REFERENCES}

Y.-C. Kan C.-K. Chen, "A Wearable Inertial Sensor Node for Body Motion Analysis," IEEE Sensors Journal, vol. 12, pp. 651-657, 2012.

D. Z. Stupar, J. S. Bajic, L. M. Manojlovic, M. P. Slankamenac, A. V. Joza, and M. B. Zivanov, "Wearable Low-Cost System for Human Joint Movements Monitoring Based on Fiber-Optic Curvature Sensor," IEEE Sensors Journal, vol. 12, pp. 3424-3431, 2012.

H. R. Chuang and W. T. Chen, "Computer simulation of the humanbody effects on a circular-loop-wire antenna for radio-pager communications at 152, 280, and $400 \mathrm{MHz}$," IEEE Transactions on Vehicular Technology, vol. 46, pp. 544-559, Aug 1997.

S. Cheng, P. Hallbjorner, A. Rydberg, D. Vanotterdijk, and P. van Engen, "T-matched dipole antenna integrated in electrically small body-worn wireless sensor node," IET Microwaves, Antennas \& Propagation, vol. 3, pp. 774-781, 2009.

D. A. James, R. I. Leadbetter, M. R. Neeli, B. J. Burkett, D. V. Thiel, and J. B. Lee, "An integrated swimming monitoring system for the biomechanical analysis of swimming strokes," Sports Technology, vol. 4, pp. 141-150, 2011/08/01 2011.

L. Xu, F. Yang, Y. Jiang, L. Zhang, C.Feng, N. Bao, "Variation of Received Signal Strength in Wireless Sensor Network," $3^{\text {rd }}$ Int. Conf. on Advanced Computer Control (ICACC), pp. 151-154, 2011.

H. Giddens, D. L. Paul, G. S. Hilton, and J. P. McGeehan, "Influence of body proximity on the efficiency of a wearable textile patch antenna," $6^{\text {th }}$ European Conference on Antennas and Propagation (EUCAP), pp. 1353-1357, 2012.

Y. Kim, H. Kim, H.-J. Yoo, "Electrical Characterization of ScreenPrinted Circuits on the Fabric," IEEE Trans. Advanced Packaging, vol. 33, pp. 196-205, 2010.

G. Mattana, T. Kinkeldei, D. Leuenberger, C. Ataman, J. J. Ruan, F. Molina-Lopez, et al., "Woven Temperature and Humidity Sensors on Flexible Plastic Substrates for E-Textile Applications," IEEE Sensors Journal, vol. 13, pp. 3901-3909, 2013.

G. A. Conway and W. G. Scanlon, "Antennas for Over-Body-Surface Communication at $2.45 \mathrm{GHz}$," IEEE Trans. on Antennas and Propagation, vol. 57, pp. 844-855, Apr 2009.

Y. Zhou, C.-C. Chen, and J. L. Volakis, "Dual Band Proximity-Fed Stacked Patch Antenna for Tri-Band GPS Applications," IEEE Trans. Antennas and Propagation, vol. 55, pp. 220-223, 2007.

C.-C. Lin, P. Jin, and R. W. Ziolkowski, "Multi-Functional, Magnetically-Coupled, Electrically Small, Near-Field Resonant Parasitic Wire Antennas," IEEE Tran. Antennas and Propagation, vol. 59, pp. 714-724, 2011.

C. L. Holloway, D. A. Hill, J. M. Ladbury, and G. Koepke, "Requirements for an effective reverberation chamber: Unloaded or loaded," IEEE Trans. Electromagnetic Compatibility, vol. 48, pp. 187-194, Feb 2006.

C. Orlenius, M. Franzen, P. S. Kildal, and U. Carlberg, "Investigation of Heavily Loaded Reverberation Chamber for Testing of Wideband Wireless Units," IEEE Antennas and Propagation Society International Symposium, pp. 3569-3572, 2006.

N. Davey, M. Anderson, and D. A. James, "Validation trial of an accelerometer-based sensor platform for swimming," Sports Technology, vol. 1, pp. 202-207, 2008.

K. Karlsson, J. Carlsson, and P. S. Kildal, "Reverberation chamber for antenna measurements: Modeling using method of moments, spectral domain techniques, and asymptote extraction," IEEE Trans. Antennas and Propagation, vol. 54, pp. 3106-3113, Nov 2006. M. Koohestani, N. Pires, A. K. Skriverviky, and A. A. Moreira, "Influence of the human body on a new coplanar-fed Ultra-Wideband antenna," $6^{\text {th }}$ European Conference on Antennas and Propagation (EUCAP), pp. 316-319, 2012.

D.Andreuccetti, R.Fossi, and C.Petrucci. Calculation of the Dielectric Properties of Body Tissues in the frequency range $10 \mathrm{~Hz}$ $100 \mathrm{GHz}$. 1997-2012. Available: niremf.ifac.cnr.it 
[19] M. Zennaro, E. Pietrosemoli, A. Bagula, and S. Nleya, "On the relevance of using affordable tools for white spaces identification," IEEE $8^{\text {th }}$ International Conference on Wireless and Mobile Computing, Networking and Communications (WiMob), pp. 606$611,2012$.

[20] R. E. Collin, Antennas and Radiowave Propagation: McGraw-Hill College, 1985. 